\title{
APLICAÇÃO DE MOLIBDÊNIO E COBALTO NA SEMENTE PARA CULTIVO DA SOJA ${ }^{(1)}$
}

\author{
JOSÉ ALFREDO PRESTES MARCONDES ${ }^{(2)}$; EDUARDO FÁVERO CAIRES ${ }^{(3,4)}$
}

\begin{abstract}
RESUMO
A aplicação de molibdênio e cobalto nas sementes tem sido uma prática comum para o cultivo de soja no Brasil. O objetivo deste trabalho foi avaliar o efeito da aplicação de molibdênio e cobalto na nodulação da soja, na nutrição da planta e seus reflexos sobre o rendimento de grãos. O experimento foi realizado no município de Ponta Grossa (PR), em Latossolo Vermelho textura argilosa, com $\mathrm{pH}\left(\mathrm{CaCl}_{2}\right.$ $\left.0,01 \mathrm{~mol} \mathrm{~L}^{-1}\right) 5,2$. O delineamento experimental empregado foi o de blocos completos ao acaso, em esquema fatorial $2 \times 4$, com quatro repetições. Os tratamentos constituíram-se de duas doses de Mo $\left(0\right.$ e $\left.48 \mathrm{~g} \mathrm{ha}^{-1}\right)$ e quatro doses de Co $\left(0,2\right.$, 4, e $\left.8 \mathrm{~g} \mathrm{ha}^{-1}\right)$, aplicadas via semente. Não se observou interação significativa entre esses dois elementos. A aplicação de molibdênio e, ou cobalto na semente de soja não alterou a nodulação e a eficiência do processo biológico de fixação de $\mathrm{N}_{2}$, avaliada pela absorção de nitrogênio e pela concentração de nitrogênio nas folhas e nos grãos. O molibdênio aplicado na semente reduziu o teor de ferro nas folhas e não alterou a produtividade da soja. Houve redução linear na altura das plantas, na concentração foliar de zinco e no rendimento de grãos de soja com as doses de cobalto aplicadas. Pelos resultados observou-se que a aplicação de molibdênio na semente de soja não é necessária em solo com $\mathrm{pH}\left(\mathrm{CaCl}_{2} 0,01 \mathrm{~mol} \mathrm{~L}^{-1}\right)$ 5,2 e que o cobalto aplicado na semente em doses superiores a 3,4 $\mathrm{g} \mathrm{ha}^{-1}$ é tóxico para a cultura da soja.
\end{abstract}

Palavras-chave: Glycine max (L.) Merrill, nodulação, fixação simbiótica, nitrogênio, nutrição mineral.

\section{ABSTRACT}

\section{APPLICATION OF MOLYBDENUM AND COBALT IN SOYBEAN SEEDS}

Molybdenum and Cobalt applied to seeds is a common practice of soybean cultivation in Brazil. A field experiment was carried out on a Clay Rhodic Hapludox soil, $\mathrm{pH}\left(0.01 \mathrm{~mol} \mathrm{~L}^{-1} \mathrm{CaCl}_{2}\right) 5.2$, in Ponta Grossa, State of Paraná, Brazil, aiming to evaluate the effects of Mo and Co seed application on soybean nodulation, mineral nutrition, and grain yield. Seed treatments with Mo $\left(0\right.$ and $\left.48 \mathrm{~g} \mathrm{ha}^{-1}\right)$ and Co $(0,2,4$ and $8 \mathrm{~g} \mathrm{ha}^{-1}$ ) were tested in randomized complete block, with four replications, in a $2 \times 4$ factorial scheme. No interaction occurred between the effects of the two elements. There was no effect of treatments on nodulation as the efficiency of $\mathrm{N}_{2}$ symbiotic fixation, evaluated by soybean $\mathrm{N}$ uptake, or content in leaf or grain. Molybdenum treatment decreased Fe content in the leaves but did not affect soybean yield. There was a significant linear decrease in plant height, leaf concentration of $\mathrm{Zn}$, and grain yield proportional to the Co amount applied. The results showed that the application of Mo is not necessary in soil with $\mathrm{pH}\left(0.01 \mathrm{~mol} \mathrm{~L}^{-1} \mathrm{CaCl}_{2}\right) 5.2$, and that $\mathrm{Co}$ applied to the seed at rates higher than $3.4 \mathrm{~g} \mathrm{ha}^{-1}$ is toxic to the plant.

Key words: Glycine $\max ($ L.) Merrill, nodulation, symbiotic fixation, nitrogen, mineral nutrition.

$\left({ }^{1}\right)$ Recebido para publicação em 23 de novembro de 2004 e aceito em 4 de agosto de 2005.

$\left(^{2}\right)$ Mestrado em Ciência do Solo - Universidade Federal do Paraná (UFPR), 80035-050 Curitiba (PR).

$\left({ }^{3}\right)$ Departamento de Ciência do Solo e Engenharia Agrícola, Universidade Estadual de Ponta Grossa (UEPG), 84030-900 Ponta Grossa (PR). E-mail: efcaires@uepg.br.

$\left({ }^{4}\right)$ Com bolsa de produtividade em pesquisa do CNPq. 


\section{INTRODUÇÃO}

A soja apresenta elevada capacidade de suprir suas necessidades nutricionais em nitrogênio por meio da fixação biológica do $\mathrm{N}_{2}$, graças ao estabelecimento da associação simbiótica entre essa leguminosa e a bactéria do gênero Bradyrhizobium, por intermédio do complexo enzimático da nitrogenase. O molibdênio faz parte da molécula da nitrogenase, que catalisa a redução do $\mathrm{N}_{2}$ atmosférico a $\mathrm{NH}_{3}$. A nitrogenase de molibdênio consiste de uma ferro-proteína (Fe-proteína) e de uma molibdênio-ferro-proteína (MoFe-proteína). A Fe-proteína funciona como doadora de elétrons para a MoFe-proteína, em um processo dependente de hidrólise de MgATP (TEIXEIRA et al., 1998). Embora a estrutura da nitrogenase seja conhecida com detalhe, o mecanismo molecular da fixação de $\mathrm{N}_{2}$ está longe de ser completamente compreendido (NunEs et al., 2003). O molibdênio também faz parte da enzima redutase de nitrato, que catalisa a redução de $\mathrm{NO}_{3}{ }^{-}$a $\mathrm{NO}_{2}{ }^{-}$(Mengel e KirKby, 2001).

As quantidades de molibdênio requeridas pelas plantas são pequenas e sua aplicação via semente constitui-se uma das formas mais práticas e eficazes de adubação. O molibdênio não tem ocasionado toxicidade ao Bradyrhizobium japonicum quando aplicado nas sementes antes do inoculante, por ocasião da semeadura da soja (CAMPO e LANTMANN, 1998). As principais fontes de molibdênio são o molibdato de sódio e de amônio, o ácido molíbdico e o trióxido de molibdênio. Para a fixação biológica do $\mathrm{N}_{2}$ em soja, essas quatro fontes de molibdênio têm sido tão úteis quanto os produtos comerciais (AlBINO e CAMPO, 2001).

As respostas da soja à adubação com molibdênio, no Brasil, têm sido variáveis. Em pesquisas realizadas em diferentes condições de solo e clima não foram observados aumentos no rendimento de grãos ou de matéria seca de soja com a aplicação de molibdênio (MASCARENHAS et al., 1973; Bellintani Neto e LAM-SÁnCHEZ, 1974; LAM-SÁnCHEZ e AWAD, 1976; GALrÃo, 1991). Entretanto, em outros trabalhos foram obtidos aumentos significativos na produtividade da soja ao molibdênio aplicado (VITTI et al., 1984; SFredo et al., 1997; Voss e PÖTTKER, 2001).

LANTMANN et al. (1989) observaram que a resposta da soja à adição de molibdênio esteve intimamente relacionada com o pH do solo, sendo as maiores respostas obtidas em solos com $\mathrm{pH}$, em $\mathrm{CaCl}_{2}$, baixo ( $<4,3$ em LRa e $<4,8$ em LEa). Existem trabalhos nos quais se constatam que tanto a aplicação de molibdênio (VitTi et al., 1984) como a calagem (MAscarenhas et al., 1990) proporcionam maior absorção de molibdênio pela cultura da soja e, conseqüentemente, maior atividade da enzima redutase de nitrato, resultando em maior teor de proteína nos grãos (LANTMANN et al., 1989; MASCARENHAS et al., 1990; SFredo et al., 1997). QuAGGIO et al. (1998) verificaram que para otimizar o rendimento de soja na carência de molibdênio, seria necessário elevar a saturação por bases do solo para 70\%. Contudo, se esse valor fosse reduzido até próximo de 50\%, seria necessária a aplicação de cerca de $50 \mathrm{~g} \mathrm{ha}^{-1}$ de molibdênio, para manter o mesmo rendimento de grãos. A calagem tem sido considerada prática eficiente para o suprimento adequado de molibdênio (Rosolem e CAIREs, 1998), por torná-lo mais disponível às plantas, em conseqüência da elevação do pH do solo, que promove liberação de íons-Mo adsorvidos na superfície dos óxidos de ferro e alumínio.

O cobalto também influencia a absorção de nitrogênio por via simbiótica porque faz parte da estrutura das vitaminas $\mathrm{B}_{12}$, necessárias à síntese de leghemoglobina, que determina a atividade dos nódulos (Somasegaran e Hoben, 1994; Mengel e Kirkby, 2001). Para o cultivo da soja no Estado do Paraná, as indicações técnicas eram para aplicação de 1 a $5 \mathrm{~g} \mathrm{ha}^{-1}$ de Co (EMBrAPA SojA, 1996) e, atualmente, recomenda-se de 2 a $3 \mathrm{~g} \mathrm{ha}^{-1}$ de Co (EMBrapA SojA, 2004) via semente, com produtos de alta solubilidade. As principais fontes de cobalto são o cloreto, o sulfato e o nitrato de cobalto. Existem no mercado diversos produtos comerciais que contém molibdênio e cobalto em concentrações variáveis, quase sempre na proporção 10:1 (Mo:Co). Apesar da importância do cobalto ao processo de fixação simbiótica do $\mathrm{N}_{2}$, existem dúvidas a respeito da necessidade de sua aplicação para se obter elevado rendimento de grãos de soja. Há evidências de respostas positivas da aplicação de cobalto na fixação biológica do $\mathrm{N}_{2}$ e na produtividade da soja quando a planta está bem suprida de molibdênio (CAMPO e HuNGRIA, 2002), mas os trabalhos da literatura não têm demonstrado que isso seja verdadeiro (GALRÃO, 1991; CAMPO e LANTMANN, 1998; SFrEDO et al., 1997). Além disso, não são bem conhecidas as doses de cobalto, aplicadas via semente, que poderiam causar efeitos tóxicos para a cultura da soja.

O presente trabalho foi realizado com o objetivo de avaliar a nodulação da soja, a absorção de nitrogênio pelas plantas, a concentração de nitrogênio nas folhas e nos grãos, a nutrição da planta e o rendimento de grãos, considerando a aplicação de molibdênio e de cobalto na semente.

\section{MATERIAL E MÉTODOS}

O experimento foi realizado no município de Ponta Grossa (PR), em um Latossolo Vermelho textura argilosa. Análises químicas do solo (PAVAn et al., 1992), da camada de $0-20 \mathrm{~cm}$, realizadas antes da 
instalação do experimento, revelaram os seguintes resultados: $\mathrm{pH}\left(\mathrm{CaCl}_{2}\right.$ 0,01 $\left.\mathrm{mol} \mathrm{L}^{-1}\right)$ 5,2; $53,5 \mathrm{mmol}_{\mathrm{c}}$ $\mathrm{dm}^{-3} \mathrm{de} \mathrm{H}^{+}+\mathrm{Al}^{3+} ; 25 \mathrm{mmol}_{\mathrm{c}} \mathrm{dm}^{-3} \mathrm{de} \mathrm{Ca}^{2+} ; 20 \mathrm{mmol}_{\mathrm{c}}$ $\mathrm{dm}^{-3}$ de $\mathrm{Mg}^{2+} ; 4,0 \mathrm{mmol}_{\mathrm{C}} \mathrm{dm}^{-3} \mathrm{de} \mathrm{K}^{+} ; 5,6 \mathrm{mg} \mathrm{dm}^{-3} \mathrm{de}$ P (Mehlich-1); $40 \mathrm{~g} \mathrm{dm}^{-3}$ de matéria orgânica e $48 \%$ de saturação por bases.

Os tratamentos, aplicados em parcelas de 10,8 $\mathrm{m}^{2}(4,0 \times 2,7 \mathrm{~m})$, foram dispostos em blocos completos ao acaso, em esquema fatorial $2 \times 4$, com quatro repetições, e constaram da aplicação de duas doses de molibdênio: 0 e $48 \mathrm{~g}_{\mathrm{g}}{ }^{-1}$ e quatro doses de cobalto: $0,2,4$, e $8 \mathrm{~g} \mathrm{ha}^{-1}$, nas sementes. A dose de molibdênio empregada foi baseada nos resultados obtidos por QuAGGio et al. (1998). As doses de cobalto foram definidas de acordo com as recomendações da EMBRAPa Soja (1996), visando-se obter resposta da soja por meio do modelo quadrático, possivelmente com efeitos benéficos em doses baixas e tóxicos em doses mais elevadas. As fontes de molibdênio e de cobalto utilizadas foram o molibdato de sódio $\left(\mathrm{Na}_{2} \mathrm{MoO}_{4} \cdot 2 \mathrm{H}_{2} \mathrm{O}\right)$ e o sulfato de cobalto $\left(\mathrm{CoSO}_{4} \cdot 7 \mathrm{H}_{2} \mathrm{O}\right)$, respectivamente.

A semeadura da soja, cultivar Coodetec 201, foi realizada em 28 de dezembro de 1999, manualmente, em sistema convencional de preparo de solo, utilizando-se 15 sementes por metro e espaçamento de $0,45 \mathrm{~m}$ entre as linhas. A adubação utilizada na semeadura foi de 330 $\mathrm{kg} \mathrm{ha}{ }^{-1} \mathrm{da}$ fórmula 0-20-10 (N- $\left.\mathrm{P}_{2} \mathrm{O}_{5}-\mathrm{K}_{2} \mathrm{O}\right)$, conforme os resultados de análise de solo (Embrapa Soja, 1996). As sementes foram submetidas à inoculação com estirpes selecionadas de Bradyrhizobium japonicum (200 g de inoculante por $50 \mathrm{~kg}$ de sementes). Utilizouse inoculante turfoso de alta qualidade, com concentração de $1,0 \times 10^{9}$ células por grama, o que equivale a, aproximadamente, 570.000 células por semente de soja. A temperatura média do ar e a precipitação pluvial ocorrida durante o ciclo da cultura foram, respectivamente, de $20^{\circ} \mathrm{C}$ e $769 \mathrm{~mm}$.

No início do florescimento da cultura foi feita amostragem de folhas, coletando-se a terceira folha a partir do ápice das plantas, em número de dez por parcela. Essas amostras foram lavadas em água desionizada, colocadas para secar em estufa com circulação forçada de ar à temperatura de $60 \pm 2{ }^{\circ} \mathrm{C}$, até atingir massa constante, e moídas. Foram determinadas as concentrações de nitrogênio, fósforo, potássio, cálcio, magnésio, enxofre, cobre, ferro, manganês e zinco, conforme os métodos descritos em Malavolta et al. (1997). Nessa época, retirou-se também, de cada parcela, um metro contínuo de plantas $\left(0,45 \mathrm{~m}^{2}\right)$ contendo raízes, à profundidade de
0-20 cm, com o auxilio de uma pá de corte. Essas amostras foram separadas em parte aérea e raízes e, após, foi determinada a altura das plantas. As raízes foram lavadas em água corrente e, depois da remoção do solo nelas aderido, os nódulos foram destacados e contados. Esses nódulos foram colocados para secar em estufa com circulação forçada de ar à temperatura de $60 \pm 2{ }^{\circ} \mathrm{C}$, até atingir massa constante, sendo então avaliada sua massa seca.

No estádio $R_{6}$ da escala de FeHr et al. (1971), aos 100 dias após a semeadura, retirou-se, de cada parcela, meio metro contínuo da parte aérea de plantas. Essas amostras foram lavadas em água desionizada, separadas em folhas, hastes e vagens, colocadas para secar em estufa com circulação forçada de ar à temperatura de $60 \pm 2{ }^{\circ} \mathrm{C}$, até atingir massa constante, e moídas. Foram avaliadas a produção de matéria seca e a concentração de nitrogênio, nas diferentes partes da planta, por meio de digestão sulfúrica e destilação pelo método Kjeldahl (Malavolta et al., 1997).

Após a maturação, a soja foi colhida e trilhada, sendo então determinado o rendimento de grãos a umidade de $130 \mathrm{~g} \mathrm{~kg}^{-1}$. Foram colhidas as três linhas centrais por $3 \mathrm{~m}$ de comprimento em cada parcela, tendo sido desprezado $0,5 \mathrm{~m}$ de cada extremidade.

A avaliação dos componentes de produção foi realizada em dez plantas por parcela na ocasião da colheita. Foram avaliados o número de vagens por planta e de grãos por vagem e a massa de 100 grãos. A concentração de nitrogênio nos grãos foi determinada em amostras colhidas em cada parcela, por meio de digestão sulfúrica e destilação pelo método Kjeldahl (Malavolta et al., 1997).

Os resultados foram submetidos às análises de variância e de regressão. Equações polinomiais foram ajustadas aos dados obtidos de acordo com as doses de cobalto, adotando-se como critério para a escolha do modelo, a magnitude dos coeficientes de determinação significativos a $5 \%$. Os efeitos da aplicação de molibdênio foram comparados pelo teste de Tukey a $5 \%$.

\section{RESULTADOS E DISCUSSÃO}

Pela análise de variância não se observou interação significativa entre as doses de molibdênio e de cobalto aplicadas nas sementes de soja para os parâmetros avaliados na planta. Por essa razão, as respostas da soja à adubação com molibdênio e cobalto foram analisadas separadamente, por meio das médias dos tratamentos. 
A aplicação de molibdênio não influenciou significativamente o número e a massa de nódulos, a produção de matéria seca, a absorção de nitrogênio e a altura das plantas de soja (Tabela 1). Esses resultados confirmam os obtidos por CAMPO e Lantmann (1998), Galrão (1991) e Albino e Campo (2001) de que a aplicação de molibdênio não afeta a nodulação da soja. CAMPo e LANTMAnN (1998) trabalharam com doses de molibdênio (molibdato de sódio) via semente $\left(4,5\right.$ e 9,0 $\mathrm{g}_{\text {ha }}{ }^{-1}$ de Mo), em três localidades do Estado do Paraná (Londrina, Ponta Grossa e Campo Mourão) onde a soja tinha sido cultivada por mais de oito anos, e verificaram que não houve efeito da aplicação de molibdênio sobre o número e a massa de nódulos por planta. GalRão (1991) constatou que a aplicação de $0,25 \mathrm{~kg} \mathrm{ha}^{-1}$ de molibdênio (molibdato de sódio) no solo com incorporação não alterou o número e a massa de nódulos por planta em quatro cultivos consecutivos de soja. Albino e Campo (2001) observaram que a aplicação de $40 \mathrm{~g} \mathrm{ha}^{-1}$ de molibdênio (molibdato de sódio) nas sementes de soja, antes do inoculante, reduziu em cerca de $15 \%$ o número de células viáveis de Bradyrhizobium por semente, em relação ao tratamento apenas inoculado, mas não influenciou o número e a massa de nódulos, bem como a extração de nitrogênio pela parte aérea das plantas.

O número de vagens por planta e de grãos por vagem, a massa de 100 grãos, o rendimento e o teor de nitrogênio nos grãos não foram influenciados de forma significativa pela aplicação de molibdênio nas sementes (Tabela 2). Em outros trabalhos também não foram observados aumentos no rendimento de grãos ou de matéria seca de soja com a aplicação de molibdênio (MASCARENHAS et al., 1973; BELLINTANI NETO e Lam-SÁnchez, 1974; Lam-Sánchez e Awad, 1976; GaLrão, 1991).

Tabela 1. Número e massa de nódulos, produção de matéria seca da parte aérea, nitrogênio absorvido e altura de plantas em função da aplicação de molibdênio na semente de soja

\begin{tabular}{|c|c|c|c|c|c|}
\hline \multirow{2}{*}{ Tratamento } & \multicolumn{2}{|c|}{ Nódulos } & \multirow{2}{*}{$\begin{array}{l}\text { Matéria seca } \\
\text { da parte aérea }\end{array}$} & \multirow{2}{*}{$\begin{array}{l}\text { Nitrogênio } \\
\text { absorvido }\end{array}$} & \multirow{2}{*}{$\begin{array}{l}\text { Altura } \\
\text { da planta }\end{array}$} \\
\hline & Número & Massa & & & \\
\hline & $\mathrm{n} \cdot{ }^{\mathrm{o}} \mathrm{m}^{-2}$ & $\mathrm{~g} \mathrm{~m}^{-2}$ & 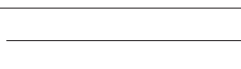 & -1 & $\mathrm{~m}$ \\
\hline Sem Mo & $2.081 \mathrm{a}$ & 9,4 a & $7.785 \mathrm{a}$ & $191 \mathrm{a}$ & 0,65 a \\
\hline Com Mo & $2.413 \mathrm{a}$ & $9,4 \mathrm{a}$ & $7.545 \mathrm{a}$ & $175 \mathrm{a}$ & $0,62 \mathrm{a}$ \\
\hline Valor F & $3,82 \mathrm{~ns}$ & $0,00 \mathrm{~ns}$ & $0,14 \mathrm{~ns}$ & $0,71 \mathrm{~ns}$ & $2,12 \mathrm{~ns}$ \\
\hline C.V. $(\%)$ & 21,3 & 18,2 & 23,5 & 28,5 & 10,4 \\
\hline
\end{tabular}

ns: não-significativo. Médias seguidas de mesma letra na coluna não diferem pelo teste de Tukey $(P=0,05)$.

Tabela 2. Número de vagens por planta e de grãos por vagem, massa de 100 grãos, concentração de nitrogênio nos grãos e rendimento de grãos em função da aplicação de molibdênio na semente de soja

\begin{tabular}{|c|c|c|c|c|c|}
\hline Tratamento & $\begin{array}{c}\text { Vagens } \\
\text { por planta }\end{array}$ & $\begin{array}{c}\text { Grãos por } \\
\text { vagem }\end{array}$ & $\begin{array}{l}\text { Massa de } \\
100 \text { grãos }\end{array}$ & $\begin{array}{c}\text { Nitrogênio } \\
\text { nos grãos }\end{array}$ & $\begin{array}{c}\text { Rendimento } \\
\text { de grãos }\end{array}$ \\
\hline & \multicolumn{2}{|c|}{ n. ${ }^{\circ}$} & $\mathrm{g}$ & $\mathrm{kg} \mathrm{ha}^{-1}$ & $\mathrm{~g} \mathrm{~kg}^{-1}$ \\
\hline Sem Mo & $39 a$ & $2,0 \mathrm{a}$ & $20,6 \mathrm{a}$ & $60 \mathrm{a}$ & $3.015 \mathrm{a}$ \\
\hline Com Mo & $40 \mathrm{a}$ & $2,1 \mathrm{a}$ & $20,8 \mathrm{a}$ & $61 \mathrm{a}$ & $3.072 \mathrm{a}$ \\
\hline Valor F & $0,63 \mathrm{~ns}$ & $0,61 \mathrm{~ns}$ & $0,34 \mathrm{~ns}$ & $0,43 \mathrm{~ns}$ & $0,71 \mathrm{~ns}$ \\
\hline C.V. $(\%)$ & 12,1 & 5,2 & 4,9 & 3,6 & 6,3 \\
\hline
\end{tabular}

ns: não-significativo. Médias seguidas de mesma letra na coluna não diferem pelo teste de Tukey $(P=0,05)$. 
A calagem aumenta o rendimento de soja (QuAGgio et al. 1982; GAllo et al., 1986; QuAGgio et al., 1998) e reduz a resposta da cultura à aplicação de molibdênio, por torná-lo mais disponível às plantas, em conseqüência da elevação do $\mathrm{pH}$ do solo (LANTAMAn et al., 1989; CAMPo e LANTMANN, 1998). QuAGGIO et al. (1998) verificaram que para solo com saturação por bases ao redor de $25 \%$, a resposta da soja foi linear até a dose de $100 \mathrm{~g} \mathrm{ha}^{-1}$ de molibdênio, com acréscimos no rendimento de grãos da ordem de $700 \mathrm{~kg} \mathrm{ha}^{-1}$, os quais foram reduzidos para $300 \mathrm{~kg} \mathrm{ha}$ 1 , quando a saturação por bases foi elevada para $45 \%$, por meio da calagem.

Os teores de nutrientes nas folhas de soja foram muito pouco influenciados pela aplicação de molibdênio nas sementes (Tabela 3). Somente a concentração foliar de ferro foi reduzida com a aplicação de molibdênio. Distúrbios no metabolismo do ferro na planta, em decorrência de relação antagônica entre Mo e Fe, têm sido descritos na literatura (Olsen, 1972; Gupta e Lipsett, 1981). Esse efeito, porém, necessita ser mais bem investigado porque QuAGGio et al. (1998) não observaram alterações nas concentrações de ferro nas folhas de soja com a aplicação de até $100 \mathrm{~g} \mathrm{ha}^{-1}$ de molibdênio nas sementes, na forma de molibdato de amônio. Cabe ressaltar que a redução do ferro no tecido foliar da soja com a aplicação de molibdênio foi significativa, mas ocorreu em pequena magnitude, e que os teores de ferro nas folhas se mantiveram em níveis considerados normais para a cultura (MALAVOLTA et al., 1997). Destaca-se ainda que a aplicação de molibdênio não influenciou a concentração de nitrogênio nas folhas de soja, o que está de acordo com os resultados obtidos por TANAKA et al. (1993) e por Campo e Lantmann (1998). As necessidades nutricionais em nitrogênio pela cultura da soja foram plenamente supridas pelo processo de fixação simbiótica do $\mathrm{N}_{2}$, independentemente da aplicação de molibdênio nas sementes.
A nodulação das plantas, a produção de matéria seca e a absorção de nitrogênio pela soja não foram alteradas com a aplicação de cobalto (Tabela 4). Ahmed e Evans (1960) obtiveram efeito positivo da adição de cobalto na nodulação e na absorção de nitrogênio pela soja cultivada em solução nutritiva. No entanto, GalRão (1991) verificou que uma única aplicação de $0,4 \mathrm{~kg} \mathrm{ha}^{-1}$ de cobalto no solo com incorporação aumentou a massa de nódulos por planta somente no quarto cultivo com soja. Em outro trabalho, a aplicação de $0,75 \mathrm{~g} \mathrm{ha}^{-1}$ de cobalto nas sementes de soja, de forma isolada ou junto com o molibdênio, não melhorou a nodulação e a absorção de nitrogênio pela cultura (CAMPO e LANTMANN, 1998). Campo e Hungria (2002) descrevem experimento em que a aplicação de 2,5 ou $5,0 \mathrm{~g} \mathrm{ha}^{-1}$ de cobalto junto com o molibdênio nas sementes de soja, não causou incremento significativo no número de nódulos por planta em comparação com a aplicação isolada de molibdênio. Por esses resultados, verifica-se que a aplicação de cobalto não tem proporcionado influência positiva no processo de fixação simbiótica do $\mathrm{N}_{2}$ para a soja cultivada em condições de campo.

A altura das plantas de soja foi reduzida linearmente com as doses de cobalto aplicadas (Tabela 4). Houve um decréscimo médio de $0,01 \mathrm{~m}$ na altura das plantas para cada grama de cobalto aplicado. A redução no crescimento das plantas de soja certamente ocorreu em função da fitotoxicidade do elemento para a cultura de acordo com as doses empregadas. No Estado do Paraná, as recomendações técnicas para a cultura da soja indicavam a aplicação de até $5 \mathrm{~g} \mathrm{ha}^{-1}$ de Co via semente (EMBrapa SojA, 1996). A dose de Co recomendada para aplicação na semente de soja foi reduzida para 2 a $3 \mathrm{~g} \mathrm{ha}^{-1}$ (Embrapa Soja, 2004) por causa de efeitos fitotóxicos do elemento. Essa redução da dose de Co recomendada para aplicação via semente em soja, devido a problemas de fitotoxicidade do elemento, concorda com os resultados obtidos no presente trabalho.

Tabela 3. Concentração de nutrientes nas folhas em função da aplicação de molibdênio na semente de soja

\begin{tabular}{|c|c|c|c|c|c|c|c|c|c|c|}
\hline Tratamento & $\mathrm{N}$ & $\mathrm{P}$ & K & $\mathrm{Ca}$ & $\mathrm{Mg}$ & S & $\mathrm{Cu}$ & $\mathrm{Fe}$ & Mn & $\mathrm{Zn}$ \\
\hline & 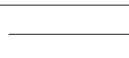 & 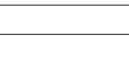 & $\mathrm{g}$ & $\mathrm{g}^{-1}$ & 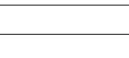 & 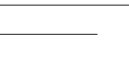 & 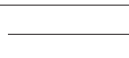 & $\mathrm{mg}$ & $\mathrm{kg}^{-1}$ & \\
\hline Sem Mo & $48,3 \mathrm{a}$ & $3,6 \mathrm{a}$ & $20,5 \mathrm{a}$ & $8,4 \mathrm{a}$ & $3,3 \mathrm{a}$ & $2,5 \mathrm{a}$ & $14,5 \mathrm{a}$ & $100,6 \mathrm{a}$ & $61,8 \mathrm{a}$ & $18,3 \mathrm{a}$ \\
\hline Com Mo & $47,4 \mathrm{a}$ & $3,4 \mathrm{a}$ & $21,2 \mathrm{a}$ & $8,4 \mathrm{a}$ & $3,3 \mathrm{a}$ & $2,4 \mathrm{a}$ & $15,1 \mathrm{a}$ & $87,4 \mathrm{~b}$ & $60,6 \mathrm{a}$ & $19,2 \mathrm{a}$ \\
\hline Valor F & $0,18 \mathrm{~ns}$ & $2,01 \mathrm{~ns}$ & $2,64 \mathrm{~ns}$ & $0,04 \mathrm{~ns}$ & $0,01 \mathrm{~ns}$ & $0,24 \mathrm{~ns}$ & $0,22 \mathrm{~ns}$ & 8,35 ** & $1,22 \mathrm{~ns}$ & $0,96 \mathrm{~ns}$ \\
\hline C.V. (\%) & 12,4 & 9,2 & 5,8 & 9,8 & 11,4 & 20,8 & 12,7 & 13,9 & 5,0 & 14,4 \\
\hline
\end{tabular}

ns: não-significativo; **: significativo a $P<0,01$. Médias seguidas de mesma letra na coluna não diferem pelo teste de Tukey $(P=0,05)$. 
Cabe destacar que a utilização de $8 \mathrm{~g} \mathrm{ha}^{-1}$ de cobalto causou sintomas visuais de toxicidade nas plantas de soja emergidas, caracterizados por clorose e necrose dos bordos das folhas primordiais.

O número de vagens por planta e de grãos por vagem, a massa de 100 grãos e o teor de nitrogênio nos grãos de soja (Tabela 5) não foram influenciados significativamente com a aplicação de cobalto nas sementes. Houve redução linear no rendimento de grãos de soja de acordo com as doses de cobalto aplicadas. Como não houve efeito positivo do cobalto em doses mais baixas, prevaleceu o seu efeito negativo em doses mais elevadas, causando redução linear na produtividade da soja. Em outros trabalhos também não foram verificadas alterações no rendimento de grãos de soja com a aplicação de cobalto no solo (GALRÃo, 1991) ou via semente (SFredo et al., 1997; CAmpo e Lantmann, 1998). A aplicação de $0,75 \mathrm{~g} \mathrm{ha}^{-1}$ de cobalto nas sementes de soja, de forma isolada ou junto com o molibdênio, não causou alteração no rendimento de grãos da cultura (CAMPO e LANTMANN, 1998). Vitтi et al. (1984) obtiveram a máxima produtividade de soja ajustada à aplicação de $236 \mathrm{~g} \mathrm{ha}^{-1}$ de Quimol nas sementes $\left(23,6\right.$ e 2,36 $\mathrm{g} \mathrm{ha}^{-1}$, respectivamente, de Mo e Co), mas não foram avaliados os efeitos isolados do molibdênio e do cobalto aplicados. CAMPO e Hungria (2002) descrevem resultados semelhantes de produtividade de soja com a aplicação de $2,5 \mathrm{~g} \mathrm{ha}^{-1}$ de cobalto $\left(3.726 \mathrm{~kg} \mathrm{ha}^{-1}\right)$ ou $5,0 \mathrm{~g} \mathrm{ha}^{-1}$ de cobalto (3.659 $\mathrm{kg} \mathrm{ha}^{-1}$ ) junto com o molibdênio nas sementes, em comparação com a aplicação isolada de molibdênio (3.617 $\left.\mathrm{kg} \mathrm{ha}^{-1}\right)$.

De acordo com a equação de regressão ajustada (Tabela 5), haveria uma redução de 5\% no rendimento de grãos (produção relativa de 95\%) com a aplicação de $3,4 \mathrm{~g} \mathrm{ha}^{-1}$ de cobalto. A redução no rendimento de soja seria de $10 \%$ (produção relativa de $90 \%$ ) com a adição de $6,8 \mathrm{~g} \mathrm{ha}^{-1}$ de cobalto. Portanto, doses de cobalto aplicadas nas sementes, na forma de sulfato de cobalto, entre 3,4 e 6,8 $\mathrm{g} \mathrm{ha}^{-1}$ poderiam ser consideradas tóxicas, e acima de $6,8 \mathrm{~g}$ ha $^{-1}$ muito tóxicas, para a cultura da soja. Esses resultados concordam com as doses de cobalto ( 2 a 3 $\mathrm{g} \mathrm{ha}^{-1}$ ) atualmente recomendadas para aplicação na semente de soja no Estado do Paraná (Embrapa Soja, 2004), no que diz respeito à toxicidade do elemento para a cultura, embora não tenha sido comprovada a necessidade de sua utilização.

Tabela 4. Equações de regressão ajustadas para número e massa de nódulos, produção de matéria seca da parte aérea, nitrogênio absorvido e altura de plantas $(\hat{y})$ em função de doses de cobalto na semente $\left(\mathrm{x}, \mathrm{g} \mathrm{ha}^{-1}\right)$ de soja

\begin{tabular}{lccc}
\hline$\hat{\mathrm{y}}$ & Unidade & Equação de regressão & $\mathrm{R}^{2}$ \\
\hline Número de nódulos & $\mathrm{n}^{\mathrm{o}} \mathrm{m}^{-2}$ & $\hat{\mathrm{y}}=\overline{\mathrm{y}}=2.247$ & \\
Massa de nódulos & $\mathrm{g} \mathrm{m}^{-2}$ & $\hat{\mathrm{y}}=\overline{\mathrm{y}}=9,4$ & - \\
Matéria seca da parte aérea & $\mathrm{kg} \mathrm{ha}^{-1}$ & $\hat{\mathrm{y}}=\overline{\mathrm{y}}=7.665$ & - \\
Nitrogênio absorvido & $\mathrm{kg} \mathrm{ha}^{-1}$ & $\hat{\mathrm{y}}=\overline{\mathrm{y}}=183$ & - \\
Altura da planta & $\mathrm{m}$ & $\hat{\mathrm{y}}=0,67-0,01 \mathrm{x}$ & $0,91^{*}$ \\
\hline
\end{tabular}

*: significativo a $\mathrm{P}<0,05$.

Tabela 5. Equações de regressão ajustadas para número de vagens por planta e de grãos por vagem, massa de 100 grãos, concentração de nitrogênio nos grãos e rendimento de grãos $(\hat{y})$ em função de doses de cobalto na semente $\left(x, g^{2} a^{-1}\right)$ de soja

\begin{tabular}{lccc}
\hline$\hat{y}$ & Unidade & Equação de regressão & $R^{2}$ \\
\hline Vagens por planta & $\mathrm{n}^{\circ}$ & $\hat{\mathrm{y}}=\overline{\mathrm{y}}=39,5$ & - \\
Grãos por vagem & $\mathrm{n} .^{0}$ & $\hat{\mathrm{y}}=\overline{\mathrm{y}}=2,0$ & - \\
Massa de 100 grãos & $\mathrm{g}$ & $\hat{\mathrm{y}}=\overline{\mathrm{y}}=20,7$ & - \\
N nos grãos & $\mathrm{g} \mathrm{kg}^{-1}$ & $\hat{\mathrm{y}}=\overline{\mathrm{y}}=60,4$ & - \\
Rendimento de grãos & $\mathrm{kg} \mathrm{ha}^{-1}$ & $\hat{\mathrm{y}}=3.206,33-46,75 \mathrm{x}$ & $0,87^{* *}$ \\
\hline
\end{tabular}

**: significativo a $\mathrm{P}<0,01$. 
Os mecanismos pelos quais a toxicidade de cobalto afeta as plantas ainda não são claramente conhecidos. Os teores de zinco nas folhas de soja foram reduzidos com a aplicação de cobalto (Tabela 6). Apesar do baixo coeficiente de determinação obtido, a redução no rendimento de grãos de soja com as doses de cobalto aplicadas (Tabela 5) pode ter sido influenciada, pelo menos em parte, pelo efeito do cobalto na redução dos teores de zinco nas folhas (Tabela 6). Liu et al. (2000) verificaram que os teores de cobre, manganês e zinco nas folhas de feijão mungo (Vigna radiata L.) não foram significativamente afetados pela adição de uma concentração não-tóxica de $0,5 \mu \mathrm{M}$ de cobalto em solução nutritiva, mas houve redução de zinco no tecido foliar das plantas em concentração de $5 \mu \mathrm{M}$ de cobalto. Os demais nutrientes não foram influenciados pela aplicação de cobalto e se mantiveram em níveis considerados normais para a cultura da soja (MAlavolta et al., 1997). A interação de cobalto com a absorção e o transporte de ferro nas plantas tem sido enfatizada em uma série de estudos (Wallace et al., 1976; Terry, 1981; Blaylock et al., 1986). O excesso de cobalto pode induzir deficiência de ferro em soja (BLAYLOCK et al., 1986), por influenciar o transporte de ferro para a parte aérea, o que não foi observado no presente trabalho. Esse efeito, no entanto, parece ser importante somente quando há restrição na disponibilidade e absorção de cálcio e ferro pelas plantas (WAllace et al., 1976; TERrY et al., 1981; LiU et al., 2000). Cabe destacar a ausência de efeito da aplicação de cobalto via semente nos teores de nitrogênio nas folhas de soja, conforme também observado por CAMPo e LANTMANN (1998) em um trabalho realizado em três localidades do Estado do Paraná (Londrina, Ponta Grossa e Campo Mourão).

Tabela 6. Equações de regressão ajustadas para concentração de nutrientes na folhas ( $\hat{y})$ em função de doses de cobalto na semente $\left(\mathrm{x}, \mathrm{g} \mathrm{ha}^{-1}\right)$ de soja

\begin{tabular}{|c|c|c|c|}
\hline$\hat{y}$ & Unidade & Equação de regressão & $\mathrm{R}^{2}$ \\
\hline $\mathrm{N}$ & $\mathrm{g} \mathrm{kg}^{-1}$ & $\hat{y}=\bar{y}=47,8$ & - \\
\hline $\mathrm{P}$ & $\mathrm{g} \mathrm{kg}^{-1}$ & $\hat{y}=\bar{y}=3,5$ & - \\
\hline K & $\mathrm{g} \mathrm{kg}^{-1}$ & $\hat{y}=\bar{y}=20,9$ & - \\
\hline $\mathrm{Ca}$ & $\mathrm{g} \mathrm{kg}^{-1}$ & $\hat{\mathrm{y}}=\overline{\mathrm{y}}=8,4$ & - \\
\hline $\mathrm{Mg}$ & $\mathrm{g} \mathrm{kg}^{-1}$ & $\hat{y}=\bar{y}=3,3$ & - \\
\hline S & $\mathrm{g} \mathrm{kg}^{-1}$ & $\hat{y}=\bar{y}=2,4$ & - \\
\hline $\mathrm{Cu}$ & $\mathrm{mg} \mathrm{kg}^{-1}$ & $\hat{y}=\bar{y}=14,9$ & - \\
\hline $\mathrm{Fe}$ & $\mathrm{mg} \mathrm{kg}^{-1}$ & $\hat{y}=\bar{y}=93,9$ & - \\
\hline $\mathrm{Mn}$ & $\mathrm{mg} \mathrm{kg}^{-1}$ & $\hat{y}=\bar{y}=61,2$ & - \\
\hline $\mathrm{Zn}$ & $\mathrm{mg} \mathrm{kg}^{-1}$ & $\hat{y}=19,90-0,32 x$ & $0,45^{*}$ \\
\hline
\end{tabular}

*: significativo a $\mathrm{P}<0,05$.

\section{CONCLUSÕES}

1. A aplicação de molibdênio na semente de soja cultivada em solo com $\mathrm{pH}\left(\mathrm{CaCl}_{2} 0,01 \mathrm{~mol} \mathrm{~L}^{-1}\right)$ 5,2 não influenciou a nodulação da planta, a concentração de nitrogênio nas folhas e nos grãos, a extração de nitrogênio pela parte aérea e o rendimento de grãos.

2. O cobalto aplicado na semente não alterou a nutrição de nitrogênio e foi tóxico para a soja em doses acima de $3,4 \mathrm{~g} \mathrm{ha}^{-1}$ porque ocasionou redução superior a $5 \%$ no rendimento de grãos.

\section{REFERÊNCIAS}

AHMED, S.; EVANS, H.J. Cobalt: A micronutrient element or the growth of soybean plants under symbiotic conditions. Soil Science, Baltimore, v.90, p.205-210, 1960.

ALBINO, U.B; CAMPO, R.J. Efeito de fontes e doses de molibdênio na sobrevivência do Bradyrhizobium e na fixação biológica de nitrogênio em soja. Pesquisa Agropecuária Brasileira, Brasília, v.36, n.3, p.527-534, 2001.

BELINTANI NETO, A.M.; LAM-SÁNCHEZ, A. Efeito de molibdênio sobre a nodulação e produção de soja (Glycine $\max ($ L.) Merrill). Científica, Jaboticabal, v.1, p.13-17, 1974. 
BLAYLOCK, A.D.; DAVIS, T.D.; JOLLEY, V.D.; WALSER, R.H. Influence of cobalt and iron on photosynthesis, chlorophyll, and nutrient concentration in regreening chlorotic tomatoes and soybeans. Journal of Plant Nutrition, New York, v.9, p.823-838, 1986.

CAMPO, R.J.; HUNGRIA, M. Importância dos micronutrientes na fixação biológica do $\mathrm{N}_{2}$. Informações Agronômicas, Piracicaba, n.98, p.6-9, 2002.

CAMPO, R.J.; LANTMANN, A.F. Efeitos de micronutrientes na fixação biológica do nitrogênio e produtividade da soja. Pesquisa Agropecuária Brasileira, Brasília, v.33, n.8, p.12451253, 1998.

EMBRAPA SOJA. Recomendações técnicas para a cultura da soja no Paraná 1996/97. Londrina: Embrapa Soja, 1996. 187p. (Documentos, 97)

EMBRAPA SOJA. Tecnologias de produção de soja: Paraná 2005. Londrina: Embrapa Soja, 2004. 224p.

FEHR, W.R.; CAVINESS, C.E.; BURMOOD, D.T.; PENNINGTON, J.S. Stage of development description for soybeans (Glycine max (L.) Merrill). Crop Science, Madison, v.11, p.929-931, 1971.

GALLO, P.B.; MASCARENHAS, H.A.A.; QUAGGIO, J.A.; BATAGLIA, O.C. Resposta diferencial de soja e sorgo à calagem. Revista Brasileira de Ciência do Solo, Campinas, v.10, p.253-258, 1986.

GALRÃO, E.Z. Micronutrientes e cobalto no rendimento da soja em solo de cerrado. Revista Brasileira de Ciência do Solo, Campinas, v.15, p.117-120, 1991.

GUPTA, U.C.; LIPSETT, J. Molybdenum in soils, plants, and animals. Advances in Agronomy, New York, v.34, p.73-115, 1981.

LAM-SÁNCHEZ, A.; AWAD, M. Efeito da simazina e do molibdênio no rendimento, conteúdo protéico e nodulação da soja (Glycine max (L.) Merrill) e da simazina no rendimento e conteúdo protéico do feijoeiro (Phaseolus vulgaris L.). Científica, Jaboticabal, v.4, p.56-58, 1976.

LANTMANN, A.F.; SFREDO, G.J.; BORKERT, C.M.; OLIVEIRA, M.C.N. Resposta da soja a molibdênio em diferentes níveis de pH do solo. Revista Brasileira de Ciência do Solo, Campinas, v.13, n.1, p.45-49, 1989.

LIU, J.; REID, R.J.; SMITH, F.A. The mechanism of cobalt toxicity in mung beans. Physiologia Plantarum, Ireland, v.110, p.104-110, 2000.

MALAVOLTA, E.; VITTI, G.C.; OLIVEIRA, S.A. Avaliação do estado nutricional das plantas: princípios e aplicações. 2.ed. Piracicaba: Associação Brasileira para Pesquisa da Potassa e do Fosfato, 1997. 319p.

MASCARENHAS, H.A.A.; KIHL, R.A.S.; NAGAI, V.; BATAGLIA, O.C. Aplicação de micronutrientes em soja cultivada em solos de cerrado. O Agronômico, Campinas, v.25, p.71-73, 1973.

MASCARENHAS, H.A.A.; TEIXEIRA, J.P.F.; NAGAI, V.; TANAKA, R.T.; GALLO, P.B.; PEREIRA, J.C.V.N.A. A calagem nos teores de óleo e de proteína em soja. Bragantia, Campinas, v.49, n.1, p.171-182, 1990.
MENGEL, K.; KIRKBY, E.A. Principles of plant nutrition. 5 ed. Dordrecht: Kluwer Academic Publishers, 2001. 849p.

NUNES, F.S.; RAIMONDI, A.C.; NIEDWIESKI, A.C. Fixação de nitrogênio: estrutura, função e modelagem bioinorgânica das nitrogenases. Química Nova, São Paulo, v.26, n.6, p.872879, 2003.

OLSEN, S.R. Micronutrient interactions. In: MORTVEDT, J.J.; GIORDANO, P.M.; LINDSAY, W.L. (Ed.). Micronutrients in Agriculture. Madison: Soil Science Society of America, 1972. p.243-264.

PAVAN, M.A.; BLOCH, M.F.; ZEMPULSKI, H.C.; MIYAZAWA, M.; ZOCOLER, D.C. Manual de análise química do solo e controle de qualidade. Londrina: Instituto Agronômico do Paraná, 1992. 38p. (Circular, 76)

QUAGGIO, J.A.; MASCARENHAS, H.A.A.; BATAGLIA, O.C. Respostas da soja à aplicação de doses crescentes de calcário em solo de cerrado. II - Efeito residual. Revista Brasileira de Ciência do Solo, Campinas, v.6, p.113-118, 1982.

QUAGGIO, J.A.; GALLO, P.B.; FURLANI, A.M.C. MASCARENHAS, H.A.A. Isoquantas de produtividade de soja e sorgo para níveis de calagem e molibdênio. Revista Brasileira de Ciência do Solo, Viçosa, v.22, n.2, p.337-344, 1998.

ROSOLEM, C.A.; CAIRES, E.F. Yield and nitrogen uptake of peanuts as affected by lime, cobalt and molybdenum. Journal of Plant Nutrition, New York, v.21, p.827-835, 1998.

SFREDO, G.J.; BORKERT, C.M.; NEPOMUCENO, A.L.; OLIVEIRA, M.C.N. Eficácia de produtos contendo micronutrientes, aplicados via semente, sobre produtividade e teores de proteína da soja. Revista Brasileira de Ciência do Solo, Campinas, v.21, p.41-45, 1997.

SOMASEGARAN, P.; HOBEN, H.J. Handbook for Rhizobia: Methods in Legume-Rhizobium Technology. New York: Springer-Verlag, 1994. 450p.

TANAKA, R.T.; MASCARENHAS, H.A.A.; BULISANI, E.A.; CAMPIDELLI, C.; DIAS, O.S. Resposta da soja ao molibdênio aplicado em solo de cerrado de baixa fertilidade. Pesquisa Agropecuária Brasileira, Brasília, v.28, n.2, p.253-256, 1993.

TEIXEIRA, K.R.S.; MARIN, V.A.; BALDANI, J.I. Nitrogenase: bioquímica do processo de FBN. Seropédica: Embrapa Agrobiologia, 1998. 25p. (Documentos, 84)

TERRY, N. Physiology of trace element toxicity and its relation to iron stress. Journal of Plant Nutrition, New York, v.3, p.561578,1981

VITTI, G.C.; FORNASIERI FILHO, D.; PEDROSO, P.A.C.; CASTRO, R.S.A. Fertilizante com molibdênio e cobalto na cultura da soja. Revista Brasileira de Ciência do Solo, Campinas, v.8, p.349-352, 1984.

VOSS, M.; PÖTTKER, D. Adubação com molibdênio em soja, na presença ou ausência de calcário aplicado na superfície do solo, em plantio direto. Ciência Rural, Santa Maria, v.31, n.5, p.787-791, 2001.

WALLACE, A.; MUELLER, R.T.; ALEXANDER, G.V. High levels of four heavy metals on the iron status of plants. Communications of Soil Science and Plant Analysis, New York, v.7, p.43-46, 1976. 